\title{
Modeling Across the Curriculum
}

\section{Prof. Peter R Turner, Clarkson University}

Currently Dean of Arts \& Sciences having previously served as Chair of Mathematics and Computer Science, and before that on the faculty at the US Naval Academy and the University of Lancaster, UK. Received both B.Sc. and Ph.D. from Sheffield University. Much of my recent scholarly activity has been in the area of STEM education focusing on preparation and retention, and on initiatives for more relevant applied mathematics education in the high school - college transitional years.

\section{Dr. Jeffrey Humpherys, Brigham Young University}

Currently a professor of Mathematics at Brigham Young University, where he has served on the faculty since 2005. He received his Ph.D. > in Mathematics from Indiana University in 2002 and was an Arnold Ross Assistant Professor at The Ohio State University from 2002-2005. Since then, he has won several grants from the National Science Foundation, including a CAREER award in 2009. His current research interests are in nonlinear and stochastic dynamical systems, numerical analysis and scientific computing, healthcare analytics, actuarial science, and network science. 


\title{
Modeling across the Curriculum: A SIAM-NSF initiative
}

\author{
Peter Turner ${ }^{1}$ and Jeffrey Humpherys ${ }^{2}$
}

\section{Introduction and Background}

The Society for Industrial and Applied Mathematics, SIAM, was awarded a second National Science Foundation grant to continue the work on increasing mathematical modeling and computational applied mathematics in high school and college curricula, and to add a thread considering the implications and possibilities in the early grades. Both workshops grew out of discussions between SIAM and NSF Education and Human Resources representatives early in 2011 on the topics of undergraduate and K-12 courses and programs, college readiness and career preparation.

The main themes of the second workshop, aptly titled, 'Modeling across the Curriculum II,' MaC II, investigated ways to increase mathematical modeling across undergraduate curricula and to develop modeling content in the K-12 educational arena. Within this context it was also important to assess college STEM readiness.

The overarching goal for both workshops and the work that results from them is to:

Engage and Keep Young People in STEM Disciplines,

from K12 through Undergraduate (and Graduate) Studies, and into the Workforce.

This objective is simply stated, but less simply achieved. Developing and implementing strategies for achieving that objective are fundamental initial steps.

The primary focus of this paper concerns the MaC activities related to the undergraduate experience. It should be emphasized that the intention is to impact all students, especially in the STEM disciplines, not just those majoring in Mathematics. Certainly students in engineering programs are an especially important sector for whom a relevant, applications and computationally focused mathematical education is desirable.

The MaC II workshop picked up where the first workshop left off in many

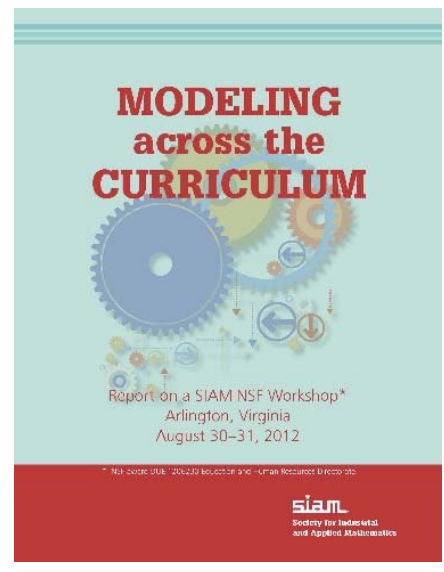
respects. The report from $\mathrm{MaC} \mathrm{I}^{1}$ is available online at www.siam.org/reports/modeling 12.pdf. The major recommendations from the first workshop can be categorized as fitting four different categories:

- $\quad$ Expand modeling in K-12

- Develop a high school one semester, or one year modeling course (with stratified content)

\footnotetext{
${ }^{1}$ School of Arts \& Sciences, Clarkson University, Potsdam NY13699-5800, pturner@clarkson.edu

2 Department of Mathematics, Brigham Young University, Provo UT 84602, jeffh@math.byu.edu
} 
- Develop modeling-based undergraduate curricula

- Develop a repository of materials for math modeling instruction and understanding.

For $\mathrm{MaC}$ II the evaluation theme of $\mathrm{MaC}$ I became an implicit requirement of all strands. The full report from $\mathrm{MaCII}^{2}$ is also available at www.siam.org/reports/modeling_14.pdf

One outcome that supports the first of these recommendations was the handbook Math Modeling: Getting Started and Getting Solutions ${ }^{3}$ which was produced by SIAM as a cooperative venture between the MaC initiative and the Moody's Mega Math Challenge, M3, which is organized by SIAM on behalf of the Moodys Foundation, m3challenge.siam.org/

While the MaC workshops are relevant at a time of growing concern about America's standards in math and science education, they were especially

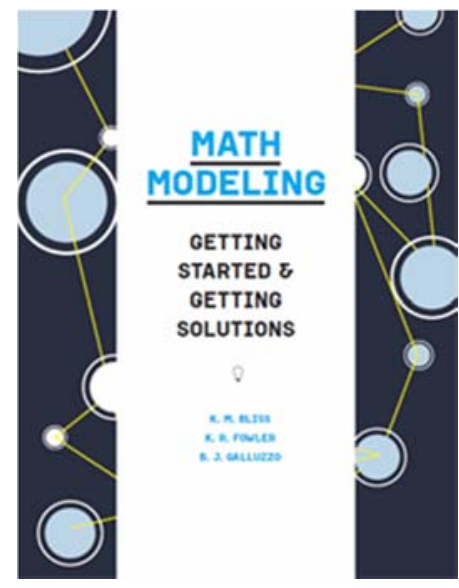
timely in the wake of the undergraduate STEM education report Engage to Excel: Producing One Million Additional College Graduates with Degrees in Science, Technology, Engineering, and Mathematics ${ }^{4}$ released by the President's Council of Advisors on Science and Technology (PCAST) in February 2012. The widespread adoption of the Common Core State Standards in Mathematics ${ }^{5}$ adds further urgency to these deliberations.

The objectives of the workshop addressed several key issues raised both in the PCAST report, such as increasing student preparedness for STEM majors and overall enhancement of STEM education in the first two years of college, and in the influential National Academies report, The Mathematical Sciences in $2025^{6}$. The results of the discussions should also help in responding to criticisms of the implementation of the Common Core State Standards and especially the recommendations to increase modeling and application-based learning in school curricula.

Mathematical modeling has the potential to increase interactions and interconnections between various STEM areas. The PCAST report, Common Core State Standards recommendations, and anecdotal information from high school and college educators call for a more
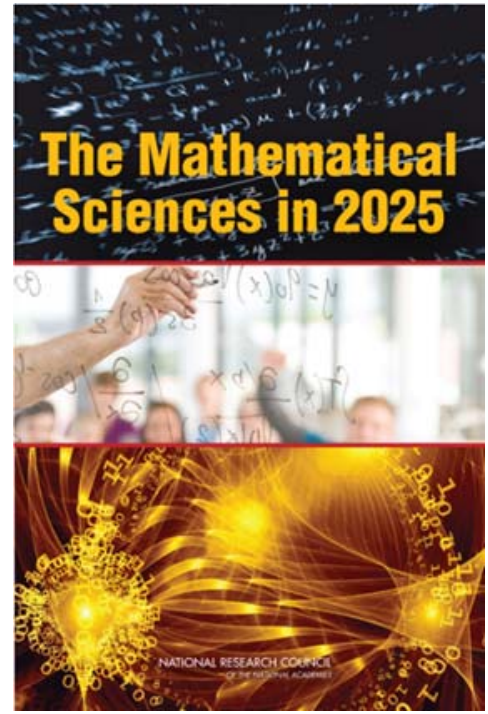
coordinated approach to STEM education. The MaC workshops have begun the process of evaluating and developing material to enhance the STEM educational spectrum in a coordinated manner. Through mathematical modeling, students in K-12 can prepare for STEM college majors and careers, thus increasing the pipeline of scientific and technical talent in America. Topical coverage should be broad in terms of both content and audience. Applied and Computational Mathematics including Statistics (ACMS) is a natural topical center for coordinated STEM programs both feeding and gaining from all other STEM fields. It should also be noted, that this preparation also serves majors in social, financial and life science majors well. This was an added theme of the recent CBMS Forum in October 2014, http://www.cbmsweb.org/Forum5/index.htm

A number of curriculum options were explored in $\mathrm{MaC}$ I and further developed in MaC II. One possible avenue is the development of undergraduate STEM degree programs as alternatives to traditional 
discipline majors. These might mirror the growth of Computational Science and Engineering programs over the past $10-15$ years, and are likely to be reflected in the growth of Data-Enabled Science and Engineering in the next several years. A key question is the extent to which mathematical modeling is treated as a stand-alone "course" or whether it should be integrated as the Modeling across the Curriculum title suggests. Coordinating the fundamental mathematics, computation, statistics and science content to support application in a wide range of STEM fields may have strong appeal to potential students.

The 2.5 day workshop was mostly comprised of active working group time. The first afternoon and early evening were plenary sessions, including an introduction from Joan Ferrini-Mundy, Assistant Director of the National Science Foundation, Education and Human Resources Division. As is noted in the undergraduate section of this report, Dr. Ferrini Mundy challenged us "to think about effective ways to educate students at the crossroads of modeling, data science, information science, computational science, and computational thinking." This is entirely consistent with the remarks in the previous paragraph.

There followed a general introduction with some background and summary of the first workshop in order to establish our starting point for MaC II discussions. This objective was furthered through a panel discussion among the three theme leaders. Topics included the following points:

- We spent a lot of time in MaC I discussing the definition of modeling. What were some of the issues and outcomes of that discussion?

- What are the differences between having a stand-alone modeling course and infusing modeling into the mathematics of different courses at different level? Can we create materials that would be flexible enough for both?

- What are our goals in trying to break up our discussions by grade level?

- What is the role of algorithms in modeling?

- What would modeling look like to a third grade class?

- What are the issues with assessment of mathematical modeling?

- What can be the role of NCTM, SIAM, ASA and other organizations in these efforts?

- The goal of this workshop is to generate not just a report, but a set of action items that a set of us will work on for the next few years. We will generate ideas for proposals related to programs, materials and training. What are potential audiences for the mini-proposals that we hope to generate from this meeting?

The afternoon plenary session included two other presentations. One was a keynote address from Mark Green on the Mathematical Sciences in $2025^{6}$ report in which he highlighted many of the common themes of that report and the Modeling across the Curriculum goals. The second was a presentation on Mathematical Modeling: Getting Started and Getting Solutions ${ }^{3}$ by Fowler and Galluzzo.

The next (main) section of this paper concentrates on the report from the undergraduate curriculum and programs working group. Of course what can be achieved in undergraduate education depends critically on the students' experiences in high school and before. Therefore brief extracts from the high school working group's findings and report are appropriate here. Among the recommendations were

1. GAIMME Report: Inspired by the ASA's GAISE Report ${ }^{7}$, we call for a report outlining Guidelines for Assessment and Instruction in Mathematical Modeling Education (GAIMME). 
2. We propose a workshop (possibly at AIM, the American Institute of Mathematics) focused on developing a high school mathematical modeling course and suggesting standards for secondary modeling education.

3. Infusion Working Group: We propose a working group of active participants be charged with formalizing strategic approaches to address challenges teachers face in infusing modeling in their daily practice.

4. Repository: We recommend a small group develop a proposal for a curated repository of modeling resources, preferably peer-reviewed. (This is likely a huge, ongoing project, needing foundation support for any chance of being successfully and popularly used by teachers and the public.)

The first of these is nearing completion, while the other three are all supported by a new NCTM-SIAM Joint Committee on Modeling across the Curriculum, which will likely form the nucleus of the coordination for the repository and play an important role in developing public awareness and the necessary professional development activities for current and future classroom teachers.

\section{Undergraduate Working Group Report}

The conference began with a charge from our opening speaker, Joan Ferrini-Mundy of the National Science Foundation, to think about effective ways to educate students at the crossroads of modeling, data science, information science, computational science, and computational thinking. After much discussion, our group identified two main pathways to help meet this goal. First, we identified the need for two different studies or reports that we thought should be commissioned to inform and educate the various stakeholders on the central role that mathematical modeling plays in society. Secondly, we suggested a greater role that SIAM and other professional organizations can and should play to help create and support communities of practitioners in applied mathematics education.

\section{Recommended Studies and Reports}

Broadly speaking we identified two primary challenges where we felt that studies or reports would be helpful and influential. The first challenge is to illustrate how mathematics connects to the rest of the world by identifying its past and current successes and articulating to STEM practitioners, and the public as a whole, the essential and centralizing role that mathematical modeling plays in innovation. The second challenge is to identify and disseminate more targeted strategies for mathematicians to attract and retain students into STEM fields through mathematical modeling.

\section{Connecting Math to Reality}

The first challenge or opportunity is about connecting mathematical modeling to the rest of the world. By looking at the world through the lens of mathematics, we see its majesty and ubiquity of mathematical modeling percolating through nearly all aspects of $21^{\text {st }}$ Century discovery and innovation. Engineers use modeling and simulation to test designs, pharmaceutical companies model drug responses and carry out adaptive clinical trials to minimize the costs and potential harm associated with testing drugs on human subjects, and markets use mathematical models to buy and sell products and services in nearly every major industry from Wall Street to Main Street.

As a working title, we suggested a report called Connecting Math to Reality, which would explore the impact that modeling has had on the world, highlighting a number of modeling achievements in history that have greatly benefited society and the world as a whole. To support this report, a series of vignettes would provide a diverse set of examples to help attract a broad readership and to provide practitioners and 
educators with examples to draw from as they communicate with the public about the mathematical sciences. Along these lines, it would be good to help educate students, teachers, guidance counselors, and parents on what modeling is and why mathematical modeling is important. In addition, we also recommended the development of some educational modules that could go with the report that could be used in classrooms. This would be particularly useful to guide the discussion on modeling given the wide adoption Common Core State Standards.

Another desired outcome of this report is to follow Ferrini-Mundy's challenge and provide the scientific community with guidance on how to educate students, largely in higher education, at the crossroads of modeling, data science, information science, computational science, and computational thinking. These disciplines are moving quickly and there are several communities, departments, and research groups that are intersecting and yet not really communicating with each other. There are concerns that artificial disciplinary silos might form and that this could be bad for science, in particular it would be bad for the students who make up the next generation of scientists. Along these lines there are concerns that these silos will use different jargon for the same ideas thus creating the need for translation in order to do interdisciplinary work. It is better to use a common language to the extent possible, and that common language should be mathematics.

In addition to the inefficiencies that can arise from this lack of cross-fertilization, it seemed desirable to stimulate cooperation, at least at the educational level, to avoid different departments teaching the same or highly similar content. With the proliferation of ideas, there's pressure to create new departments within universities, thus taxing the administrative overhead and making institutions fractured and top heavy.

It has been suggested that applied and computational mathematics has an opportunity to be the glue that connects these fields together, to help facilitate cross-fertilization, but in order to do so, substantial curricular and cultural changes will be necessary.

\section{Modeling and the Pipeline}

It is dangerous to learn to fly while flying---mistakes come at a very high cost! To avoid this, we have flight simulators that allow pilots and trainees to make mistakes virtually and learn from them without having to experience the tragedies that come from real mistakes. Similarly, it is dangerous to test out new products, services, designs, and policies in a real-world setting, such as a business or government agency, without first testing ideas in the virtual world.

Of course a major difference between a flight simulator and a market simulator is that the flight simulator has laws of physics that govern the dynamics of the airplane and allow the simulator to be nearly perfect in its representation of actual flight. In business or government, however, natural laws are replaced by market responses from both consumers and competitors, and so models tend to be complex, incremental, and uncertain instead of absolute and largely well understood. Indeed there is often a large gap between theory and practice when human interaction is concerned, and in many cases there isn't even a reasonable theory. Nonetheless, the idea is the same. Virtual experimentation is replacing many aspects of realworld implementation and the demand for modelers is rapidly increasing.

This demand translates into jobs, and so our second recommended report or study is to find ways to attract and retain students into STEM fields through mathematical modeling - we need to study and understand the STEM pipeline and the role that modeling plays, or can play, to stimulate growth and 
vibrancy in the quantitative disciplines. With the projected future shortfall of STEM graduates as described in Engage to Excel and the call for a 34\% increase in STEM majors, we see an opportunity to strengthen the pipeline through mathematical modeling, and that opportunity needs to be studied and reported to the community.

The information age has provided us with both massive amount of data and substantial computational resources whereby we can extract useful information. The computational and data sciences are a hot area and companies are clamoring to find people who can innovate in a data-rich environment. There are substantial opportunities for the mathematical community to attract and retain students if we can adapt to this growing opportunity.

One question that was raised in the workshop was whether there are other entrances into the mathematical sciences that follow an alternative track different than the usual calculus approach. Could a freshman math modeling class bring students into applied mathematics who might not otherwise be majors? Moreover, with the calculus track, is there a new approach that would improve educational outcomes? These questions should be addressed in this study.

\section{Recommendations for Professional Organizations}

In addition to the two reports or studies described above, our group identified opportunities for professional organizations to create and support communities of practitioners in applied mathematics education.

\section{SIAG on Applied Math Education}

One of the group's recommendations was that SIAM create an activity group on Applied Math Education. This would provide numerous opportunities for cooperation, collaboration, and recognition. Examples include conferences, sessions at the annual meeting, email lists, SIAM-backed blogging, and even perhaps an online magazine. There could also be awards given to departments and individuals recognizing their contributions.

Another benefit of an activity group would be the ability to pull people together to serve the community in a coherent and cohesive way. There was great interest in our group in having a curated library of trusted resources, with ratings, moderation, reviews, and ample metadata, e.g., synopsis, categorize by area, pedagogical type, review of literature, and reviewed resources, so that people can find reliable resources to use in their classrooms and even participate in the development efforts if desired. This would open substantial opportunities for both collaboration and dissemination. There are also great opportunities for social networking, tweeting, blogging, etc., to further stimulate collaboration and cooperation, and volunteers within the activity group might make good moderators and reviewers for such content.

There was some discussion on how to differentiate the activity group from SIAM's education committee. The underlying maxims guiding who does what seemed to fall on the activity group existing to support research and education activities surrounding efforts in academia, whereas the Education Committee will serve the SIAM community as a whole. For example, SIURO will be managed by the SIAM Education Committee, but a conference on Applied Math Education would be run by the activity group. 


\section{SIAM Education Committee Opportunities}

Our group also identified a need for cooperation across professional organizations and it was recommended that the SIAM Education Committee continue to make and establish connections with the education VPs of other societies and organizations. Examples of organizations should certainly include MAA, ASEE, ASA, NCTM, AMTE, COMAP, AMATYC to name a few. It would also be helpful to connect to centers that are modeling-friendly such as DIMACS, Cause, etc.

By connecting with these organizations, there's an opportunity to address a number of important questions and try to get some consensus around some of the larger educational issues in the broader STEM community. For example, what are the best practices in accessing and evaluating educational programs dealing with modeling? How does one judge creativity and higher-order thinking, how does one judge the quality of a program, the learning outcomes, program outcomes, etc.? What are the goals of a good modeling program? What does it mean to be college ready? What are the best ways to remediate? How can these groups work together to achieve better outcomes?

\section{Key Discussion Points}

The following are some discussion points that emerged from our meeting. While we did not want to make any specific recommendations in these areas, we felt that it would be worth considering the problems in our discipline and the trends that can be observed in academia and industry.

- The mathematics community is largely unaware of how math is used in other quantitative disciplines. The math curriculum has not changed much since the 1960s, and yet other related disciplines have changed substantially, and so we are really out of touch (speaking broadly not individually).

o As an example, the singular-value decomposition (SVD) in linear algebra is a widely used technique in statistics, computer science, engineering, finance, and economics, and yet many pure mathematicians are unfamiliar with the topic, in large part because good numerical algorithms weren't developed until the 1960's and 1970s. To many mathematicians, linear algebra is the study of the algebraic properties of vector spaces and linear transformations. Some mathematicians pay little attention to the geometric and operator-theoretic properties of the field where applications are most prevalent. As so many other disciplines use the SVD, it is not only important that mathematicians understand what it is, but also teach it thoroughly in linear algebra and matrix analysis courses.

o As another example, the mathematics community is largely unaware of what Bayesian Statistics is and the role that it plays in emerging fields such as machine learning and natural language processing. Latent Dirichlet Allocation (LDA) was specifically mentioned as a new idea in applied Bayesian statistics. This and other related techniques are generative modeling methods that are quite powerful.

o Compressed sensing was also mentioned as a new hot area in computational harmonic analysis. How should it and other new and emerging areas of applied mathematics be woven into the curriculum so that students learn these methods and can apply them when they get into the workforce? Even at the undergraduate level, compressed sensing could be introduced alongside 11-regularization so that problems where sparse solutions are wanted can be obtained. 
- The traditional undergraduate degree in mathematics does not prepare students for careers in industry. There are very few topics, if any, that are traditionally covered in mathematics that were developed after 1900. As a result, graduates in mathematics have few qualifications and little preparation in the workforce unless they seek (usually on their own) a background in computer programing or statistical modeling. Without these skills, math majors struggle to get the high-paying jobs that related STEM graduates get.

- It's time to grow up: There was discussion surrounding the idea that our way of life (for our discipline) can't persist if we continue to fail to connect to other disciplines and provide students with the mathematics that they need to succeed in the workforce. Over time, if we don't change, we will have resources redirected away from us. One of the participants said, "It's time to grow up".

- What algorithms should be taught in the undergraduate curriculum? In a traditional curriculum, in Calculus, Newton's and Simpson's Rule are usually taught for one-dimensional problems and Euclid's division algorithm is taught in abstract algebra. Many will struggle to come up with examples beyond that. Below are families of algorithms that are accessible to undergraduates and that could be considered at some level in a modern curriculum:

o Encryption algorithms: finding pseudo primes with Fermat's little theorem, RSA, DiffieHellman key exchange

o Solving linear systems: Jacobi, Gauss Seidel, Successive Over-Relaxation (SOR), Krylov methods such as GMRES

o Signal processing and time-series analysis algorithms: DFT, FFT, ARMA, ARIMA

o Compression algorithms: Huffman encoding, wavelets, LZW

o Tree search algorithms: AVL trees, BW-trees, B-trees

o Constrained optimization: simplex algorithm, interior-point methods

o Unconstrained optimization: Newton's method, conjugate-gradient, quasi-Newton methods

o Markov-Chain Monte Carlo Methods: Gibbs Sampling, Metropolis-Hastings, Metropolis

o Matrix Decompositions: SVD, QR, LU

o Graph Algorithms: MST, TSP, BFS, DFS, greedy algorithms

o Markov Decision Processes: multi-armed bandit problems

o State Estimation: recursive least squares, Kalman filtering, particle filters

o ODE Solvers: Runge-Kutta, boundary-value solvers

o PDE Solvers: Finite-element and finite-difference methods

- Topics that were discussed and recommended that could/should be included into the curriculum are:

o Design, analysis, and optimization of algorithms

o Probability and stochastic processes

o Bayesian statistics, machine learning, and data analytics

o Dynamical systems and Control Theory

o Optimization

- Technical Skills: Students should know how to work with data. Web scraping, regular expressions, relational databases. Additionally students who are good with scientific visualization, low-level programming $(\mathrm{C} / \mathrm{C}++)$, high-level scripting (Python, $\mathrm{R})$, and distributed 
computing technologies (such as MPI, Hadoop/Map-Reduce) will have a substantial advantage in the workforce.

\section{Conclusions and Recommendations}

The report on $\mathrm{MaC}$ I stated that "The most obvious conclusion to draw from the foregoing is perhaps that this short workshop could do little more than scratch the surface ..." in the development, recruitment, retention and education of a strong pool of STEM undergraduate majors. One of the key recommendations was that:

"There should be a follow-up workshop of longer duration which can explore questions raised in this report, the linkages between the different themes, and reach greater specificity on research questions.

- A minimum of 2.5 days seems appropriate, with

- Expanded participation including many of the attendees from the first workshop, and including

- Both pairwise and three-way interactions among themes to explore connections.

An important outcome of the second workshop will be to identify small leadership teams for each theme. The workshop steering committee would begin that process in the planning stage."

The foregoing demonstrates that this objective was met. Substantial progress was made in each of the three groups resulting in specific recommendations and action items. One overarching recommendation was again that there should be a third such workshop to address some of these specifics, and to disseminate progress that has been made in the interim.

A key recommendation from the undergraduate group, and endorsed by each of the others bears repetition here:

\section{Recommendation 1}

One of the group's recommendations was that SIAM create an activity group on Applied Math Education. This would provide numerous opportunities for cooperation, collaboration, and recognition. Examples include conferences, sessions at the annual meeting, email lists, SIAM-backed blogging, and even perhaps an online magazine. There could also be awards given to departments and individuals recognizing their contributions.

In fact this recommendation has already been implemented. SIAM Board and Council approved the establishment of SIAG/ED at their meetings in July 2014 and the group begins operation officially on January 1, 2015. It is hoped that the first SIAM Conference on Applied Math Education (September 30 October2, 2016 in Philadelphia) will coincide with the third MaC Workshop, subtitled BIG-MaC to stress the connections with Business, Industry and Government. This should also help to bring more people into the effort and facilitate both the dissemination and further development mentioned above.

Several specific suggestions from the different groups are included in the Executive Summary at the beginning of the report. 
Each of the thematic discussions was very fruitful. Important topics were identified as recommendations or action items by each group.

The early grades recommendations centered around the need for professional development and preservice training for teachers who have typically little awareness of mathematical modeling. The group developed a lengthy list of Action Items which can perhaps be summarized as

\section{Recommendation 2}

Develop strong professional development and teacher training programs, materials and support networks to provide experience, understanding and skills in mathematical modeling at levels appropriate for use in early grades classrooms.

This is a major undertaking. It probably requires the creation of some specialist teachers even for the early grades. Materials that would be needed include:

- Producing materials, including classroom posters, videos, materials for teacher training and professional development, released standardized test items and classroom projects that help communicate what mathematical modeling is.

- Developing professional development programs that train teachers (and perhaps also math specialists, district leaders, mathematicians and parents) how to do mathematical modeling and facilitate mathematical modeling for early grades.

- Creating peer-networks and social networking sites for teachers to share ideas, and locate materials that have been class tested. This site should include "promotional" videos perhaps of discussions with, or interviews of, experts and teachers to explain the modeling process and its importance.

The third Modeling across the Curriculum workshop and SIAG/ED conference should help with disseminating progress to date and advancing these goals. This recommendation clearly also necessitates involvement of teacher educators, supervisors of mathematics and mathematics education expertise. That would represent a significant broadening of the $\mathrm{MaC}$ initiative.

The High School working group made several recommendations which can be summarized in terms similar to the Early Grades' Recommendation 2 above:

\section{Recommendation 3}

Develop strong professional development programs, curricular and assessment materials, and develop working groups to investigate different strategies for introducing modeling into the high school. Some specifics components are

- $\quad$ Produce Guidelines for Assessment and Instruction in Mathematical Modeling Education (GAIMME) along the lines of the ASA's GAISE Report.

- Propose and run an American Institute of Mathematics Workshop focused on developing a high school mathematical modeling course and standards for secondary modeling education.

- Create Working Groups to study different strategies such as Infusion of Modeling into high 
school curricula, Professional Development for teachers to improve or develop their expertise, and Assessment

- Develop a curated Repository of peer-reviewed and tested materials covering projects, curricular components, career and public awareness

As in the first proposal, there is already progress to report. SIAM and the Consortium for Mathematics and its Applications, COMAP, have agreed to fund a workshop specifically charged with developing a GAIMME report.

Recommendation 1 above originated with the undergraduate curriculum group and was quickly endorsed by the other two groups. The Undergraduate section of the report also calls for two major reports which would be valuable throughout the educational continuum.

\section{Recommendation 4}

Two major reports similar to those produced for the National Academies should be commissioned:

- Connecting Mathematics to Reality, and

- Modeling and the Pipeline

The first will have value to educators, students and advisors at all levels. In particular it will arm teachers with answers to the "Why do I need to learn this?" or "When will I ever use this?" questions. Note that the way in which the second of these is worded almost pleads for an applications and modeling perspective to mathematical education. The second proposed report speaks to the vital role mathematical sciences play in the development of an appropriately prepared and skilled workforce.

It is plain from the report that much work still needs to be done. Much of this work can continue among the various teams and communities that have developed. There will be a need for a periodic reconvening of a more general group and so a third Modeling across the Curriculum workshop should be planned. Combining it with the first biennial conference of the new SIAM Activity Group in Applied Mathematics Education will enable the continued dialogue among the interested groups and the broadened participation that the conference would facilitate.

\section{References}

[1] SIAM Modeling across the Curriculum: Report on a SIAM-NSF Workshop, Society for Industrial and Applied Mathematics, SIAM, Philadelphia, 2012

[2] SIAM Modeling across the Curriculum II: Report on the second SIAM-NSF Workshop, Society for Industrial and Applied Mathematics, SIAM, Philadelphia, 2014

[3] K.M. Bliss, K.R. Fowler \& B.J. Galluzzo, Math Modeling: Getting Started and Getting Solutions, SIAM, Philadelphia, 2014

[4] President's Council of Advisors on Science and Technology, PCAST, Engage to Excel: Producing One Million Additional College Graduates with Degrees in Science, Technology, Engineering, and Mathematics, Office of Science and Technology Policy, Washington DC, February 2012, http://www.whitehouse.gov/sites/default/files/microsites/ostp/pcast-engage-to-excel-final 2-2512.pdf 
[5] Common Core State Standards Initiative, Common Core State Standards: Mathematics, http://www.corestandards.org/the-standards/mathematics \&

http://www.corestandards.org/Math/Practice

[6] National Research Council, The Mathematical Sciences in 2025, National Academies Press, Washington DC, 2013

[7] American Statistical Association, Guidelines for Assessment and Instruction in Statistics Education (GAISE) Report, American Statistical Association, Alexandria VA, 2005 